\title{
The Value of Learning and Interact Action Based on Problem Context in Network Virtual Environment
}

\author{
$\mathrm{Bo} \mathrm{Li}^{*}$ \\ Faculty of Art and Design, Xi'an University of Technology, Xi'an, Shaanxi, China
}

\begin{abstract}
At present, the network learning resource architecture mostly stays in the category of individual learning, focusing only on the listing and display of knowledge, but rarely to reflect the problem context, it is difficult to motivate learners' active exploration and learning interaction. This paper aims at the analysis of learning and interaction activities based on the problem context, realizes the construction of learning and interaction level model caused by the problem situation, and reveals the five interaction levels of the model from low level to high level. Further more, to discuss the research value of problem-based learning and interactive behaviour in the network virtual environment by using the teaching case of the interactive simulation teaching system of aircraft virtual assembly and driving operation.
\end{abstract}

\section{Introduction}

It has been 30 years since American scholar Neil first proposed the concept of "virtual environment" in 1989. Domestic and abroad scholars keep doing the things to enrich and deepen the definition of it. In a narrow sense, a virtual environment refers to the three-dimensional world in which users can integrate and experience their own existence under the user-friendly virtual technology interface, which is mainly composed of the interface of "computer + virtual technology". In a broad sense, it refers to the computerized world using virtual technology, which is mainly composed of multiple computers, virtual technology interface and network technology, such as local area network, World Wide Web (WWW), etc.

During the use of virtual reality technology, users can browse virtual objects in virtual space, and the virtual environment created by the application of virtual reality technology can make users feel their own existence environment and activities. Virtual reality is based on computer technology to create and experience the virtual world, which can contain a variety of information during the practical application, and can give full play to human perceptual and rational cognition in this multidimensional space.

(1) Immersion: the "virtual" concept of virtual reality technology is mainly because the presented environment does not exist in reality, only the virtual world created by computer technology, so users will be immersed in the virtual space. The immersion mentioned here is mainly that users use the virtual environment equipment to integrate themselves with the virtual environment, so that they are in the virtual environment. In the virtual world, users can feel and apply different objects, which is similar to the feeling of the real world. In the virtual environment, users can feel things with high authenticity and fully give them the feeling of being in the real world.

(2) Interactivity: interactivity mainly refers to the user's operation of objects in the virtual world, which can get feedback from the environment, such as communicating with others in the real life environment. The human-computer education of the virtual reality system is close to the natural interaction mode, and the user can comprehensively inspect or operate the people and objects in the virtual world through his own language, body movement and other skills The computer can adjust the sound and image of the system according to the user's instructions and actions

(3) Multi perception: the intelligent logic analysis ability possessed by the virtual reality equipment is called multi perception. In the virtual reality system, vision sensing device, hearing sensing device, touch sensing device and motion sensing device are installed, so the user can obtain different perception such as touch, hearing, vision and kinesthetic sense by using the human-computer interaction mode in the virtual environment, so as to achieve personal experience the feeling of its environment.

(4) Conceivability: through the virtual reality system, people and things that do not exist in real life can be created, and can stay in people's thoughts. Users can apply a variety of activities in the virtual environment to obtain corresponding knowledge, so that they can deeply understand and master the relevant knowledge system, generate more creative ideas, and realize a new transformation of people's cognition and thinking mode Change.

The prosperity of virtual environment not only profoundly changes people's living conditions and leisure ways, but also brings technological convenience for people to learn knowledge and explore experiments. 
Virtual learning with the help of virtual environment has become an inevitable trend of learning methods.

\section{Problem Context in virtual environment}

The basic unit of modern curriculum is problem. The main task of curriculum reform is to reorganize curriculum, that is, to organize curriculum content through problem design. It is the direction of teaching design research and practice to pay attention to problem solving and the development of learners' cognitive ability. Defining the problem can make the organization of learning activities surpass the randomness that always exists. Through the media function of problems, it can greatly promote the deep development of learners' cognitive ability.

The network virtual environment is generated by the use of computer network and graphic display technology. It has realistic three-dimensional visual, listening and other sensory and aesthetic effects. People can directly or through appropriate devices intervene in it, and have a variety of rich experience and interaction with it, including landscape, flowers and plants, architectural monuments, animals and monsters, virtual characters and communities.

Experimental psychologist Treichler has done an experiment on the main way for human to obtain information sources. Research has confirmed that $83 \%$ of the information obtained by human comes from vision and $11 \%$ from hearing. In other words, the amount of information obtained by stimulation of multiple senses is larger and more valuable than that obtained by a single sense. With the help of more and more advanced 3D engines, the problem situations presented in virtual environment are more and more gorgeous, lifelike and complex, full of extremely rich visual and auditory elements, which can present macro and micro things or scenes that are difficult to be observed or reproduced in reality. It seems to be the infinite extension or expansion of human senses in space and time. Being in such a virtual environment, it can not only obtain strong visual and auditory perception, but also trigger various imagination, association or thinking sparks, thus forming the basis for learners' continuous cognition and learning.

For the problem situation and related research in virtual environment, American scholars started relatively early. Generally speaking, situation is the integration of physics and information of problem or task. It usually includes two fields: real and virtual. Learning situation mainly refers to the challenging, interesting, real simulation that learners encounter in the virtual environment and the adaptability to the new scene which is inconsistent with their original experience.

These problem situations are the inducing factors to attract learners to immerse themselves in the deep virtual experience. The problem-solving process is a kind of thinking expansion and informal learning activity, which contains rich learning and interactive behaviour, and has important research value.

\section{Analysis of learning and interaction based on problem context}

More and more learning scientists believe that learning is not only a change in the mind of learners, but also related to the real situation (including reality and virtual); learning is not only a personal cognitive activity, it can't be separated from the learning situation and social interaction in the process. Generally speaking, learning in the network age is an organic integration and interdependence among learning, cognition and situation, which constitutes a complete and dynamic new learning map.

\subsection{An analysis of interactive activities derived from inquiry}

The exploration of many problem situations in virtual environment is often associated with a large number of tools that can be used, manipulated, combined or even processed. These items have different properties and use values, and usually need to learn to master their use methods. There are three levels of such interactive activities: the first level is simple imitation, that is, understanding, imitation and operation of general objects; the second level is understanding and processing, which is manifested in the skillful use and assembly of existing objects on the basis of mastering performance, sometimes using existing tools for operation and combination to form a new object that can solve problems; the third level is cognitive construction, It is mainly manifested in being able to flexibly use various tools to solve various challenges and problems according to specific requirements or within the specified time, such as mastering the performance and switch of various complex instruments and instruments in the highly lifelike cockpit, making the aircraft take off smoothly, etc.

\subsection{An analysis of virtual learning style from problem-orientation}

Create a good problem context and network exploration environment, and cultivate learners' analytical cognitive ability. Problem design in problem-orientation learning is a key variable that affects the effectiveness of curriculum. Learners are the subjects of virtual learning process, problem solvers and meaning constructors. A good problem situation can stimulate the enthusiasm of learners to take part in learning actively, so that learners can continuously improve their abilities in all aspects, especially the ability of thinking analysis in the process of learning knowledge and skills. Learners put forward research questions by analyzing the problem context in virtual learning course, including imagination, query, divergent thinking, etc. In this process, learners' analysis of problem context is very important and can cultivate their analytical ability.

Virtual learning course provides more opportunities for learners to independently arrange learning tasks and plans according to their actual situation, to independently 
search and collect network resources under the guidance of problems, and to get more help and guidance through interaction and cooperation with team members, so as to obtain more ways and strategies to solve problems.

\subsection{An analysis of virtual learning style from cooperative learning}

This problem-based learning method in virtual environment is the change of learners through extensive interaction with other people and environment in reality or in virtual environment, around the clarity, discussion and solution of problems. It has four characteristics: First, it is interesting, fully inherits, draws lessons from and integrates the mechanism, rules and evaluation methods of traditional games through digital and virtual representation, making the process of experience or problem exploration full of tension, stimulation and interest from the beginning to the end; Second, it is practical, requiring learners to use their brains and more hands-on, so that learners can realize the "outside" in the virtual environment It is a kind of transformation of things, and its experience and feelings tend to be true and real-time feedback, and the process is also controlled by itself; Thirdly, it is consultative, which provides learners with rich dynamic resources, information and technical support, encourages them to explore, select, process and internalize independently, and encourages and supports learners with different learning styles to enrich around specific problems There are substantive dialogues, exchanges and consultations, which produce new results in consultation and interaction. Fourthly, the field is a new open and dynamic virtual environment, a "micro version" of the real world, and an open and diverse ecology of information, cognition and learning field.

\subsection{An analysis of virtual learning style from reflection and evaluation}

Pay attention to the reflection and evaluation of the learning process. In the evaluation stage of curriculum learning, learners compare their problem-solving process with that of an expert or other students, reflect on the knowledge constructed in the process of problem-solving, and systematically and conceptually organize and integrate the knowledge. Then, the learner constructs a model about the specific problem solving process to enlighten his own problem solving and task completion process. Pay attention to the evaluation of learners to solve real problems, and make a comparative reflection of solutions and effects, and provide real-time guidance feedback. The design of the whole virtual course is problem oriented and focuses on the development of learners' cognitive ability. In the course design, it provides the opportunity of situational experience and problem-solving. Through rich case study, the learners can learn to explore and think in depth independently. The role of teachers in the whole learning process is not the decision-maker, but the guide, organizer and designer of learning materials. They are responsible for guiding learners from "Knowledge", "comprehension", "application" gradually develop to "analysis", "synthesis" and "evaluation" of knowledge.

\section{A hierarchical model of learning and interaction triggered by problem situations}

There are two important functions of problem-based cognitive style and learning interaction in virtual environment: one is to form and construct learning tasks, which have meaningful connection with learners' original cognition and experience; the other is to effectively promote frequent collision and connection between learners' knowledge, skills and experience, and generate meaningful and creative learning. According to the learning theory of constructivism, it has five attributes: constructive (exploration and knowledge construction with task goal as the core), active (purposeful activities and interactions), intentional (various tasks pointing to the goal), real (complex and vivid 3D scenes, animation, etc.), cooperative (extensive interaction and cooperation with other partners). Because this kind of learning and interaction fully embodies the activity track of "intention $\rightarrow$ action $\rightarrow$ reflection $\rightarrow$ practice", and has the power, supply and energy of continuous circulation and spiral rise, it can't be completed on one plane, but has multilevel.

Here, many learners also form a virtual learning community. There are interactions between teachers and learners, between learners and problem situations, and between learners. There are also interaction levels from low level to high level.

It is not hard to see that they are a relationship of continuous progress and integration. The first three are characterized by cognition and learning, and they are shallow level interaction. The second two are more innovative based on cognition and learning, which belong to a deep level interaction. Among them, the fifth level is the formation process of virtual learning community, which has some basic characteristics of learning community. As Leif and Wenger have pointed out, the key feature of the learning community is that there is a learning culture in which everyone can participate in the efforts to improve collective understanding. It must have four characteristics: members have different knowledge and are willing to contribute; the common goal of continuously improving collective knowledge and skills; the emphasis on learning methods; and the mechanism of sharing learned knowledge.

It undoubtedly proves the essence of learning in the Internet Era: first, learning is the process of making meaning, not the transmission of knowledge; second, learning is a meaningful dialogue, which is both internal and social consultation; third, the making or value of meaning is influenced by the community, which is not only produced in the brain, but also in the extensive interaction between individuals and the community of practice. 


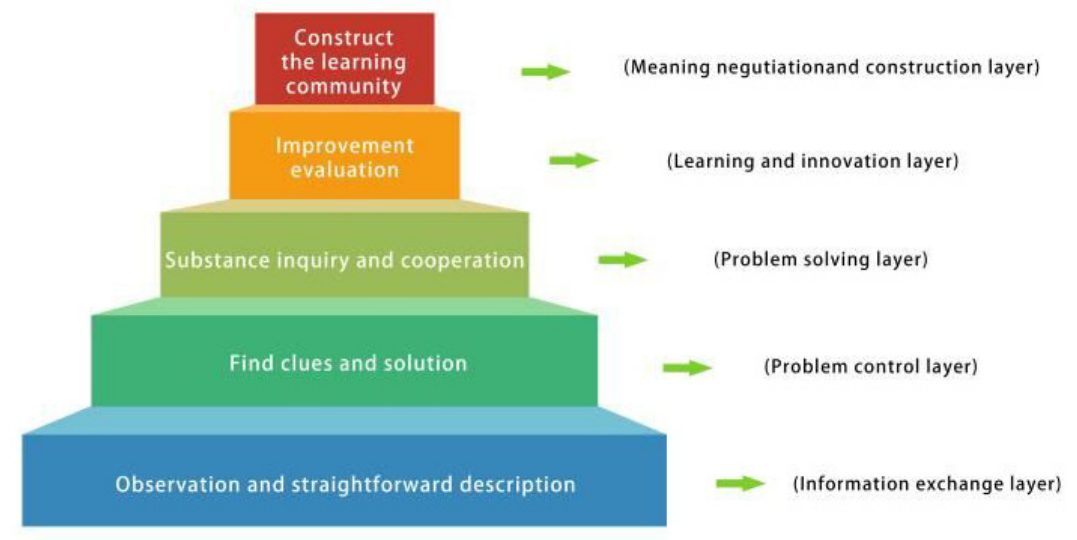

Figure 1. Problem context leads to the five Layer

\section{Case Study}

Based on the development of unity 3D and visual studio C\#, VR technology, network technology and advanced teaching methods are introduced into the virtual simulation experiment teaching. An integrated experiment platform of learning, roaming and evaluation is established. Combined with the traditional experiment teaching, an open reality which can be fully shared by teachers and students is created Test teaching resources make up for the lack of reform and innovation in teaching methods, low interest of students in learning, lack of high-quality teaching resources and other problems.

This system mainly includes computer software and hardware system, sensor equipment and so on. Among them, hardware system mainly includes the large computer system, and consists of many small computers and workstations, etc. The software system mainly includes the relevant computer modules, including the control module, the feedback module, detection module and sensor module. The control module mainly uses different information data to control the sensor, which can ensure the interaction between the user, the virtual environment and the real environment; the feedback module mainly receives the information from the sensor, there are many types of feedback sensors, which are important information interactive devices. The detection module is mainly used to detect the commands issued by users in virtual environment. Fig. 2 is a schematic diagram of the system.

The system adopts level of detail (LOD), layered call, baking and other technologies. One is to balance the virtual scene modelling to ensure the minimum model file with high-quality picture effect; the other is to realize the intelligent loading of scene model file network to ensure the smooth operation of the system. At the same time, all kinds of VR devices are used to realize the full immersion experience of $3 \mathrm{D}$ virtual scene and realize the functional requirements and effects of the virtual system.

Teaching function design: the system uses voice teaching and graphic teaching to cooperate with the cognition and learning of virtual scene. There are two ways of speech teaching in the system: one is to trigger speech by collision. Considering the immersion of virtual reality software, a voice collision retrieval switch is set. When roaming to the switch boundary, the system will automatically turn on the voice teaching switch. This way is suitable for use in a wider space, and will not cause misjudgment of triggering. Second, click to trigger the voice in the assembly workshop and the denser cabin equipment, and click the voice teaching button to open the voice teaching content. The graphic teaching method is mainly to click the button, equipment and logo to pop up the learning dialog box.

User experience design: in order to enhance the realistic experience during roaming, the first person perspective roaming function of aircraft virtual assembly and driving operation simulation system is developed based on unity $3 \mathrm{~d}$. When students operate the simulation system, they can control the roaming direction and perspective, as well as forward, turn, squat, climb, jump and other actions according to their own needs. The system interacts with the outside world in many ways, i.e. using mouse and keyboard to operate on the Internet through ordinary computer chain, or using virtual peripherals such as virtual helmet, handle and treadmill to completely "immerse" in the virtual system, so as to obtain more realistic virtual reality experience. 


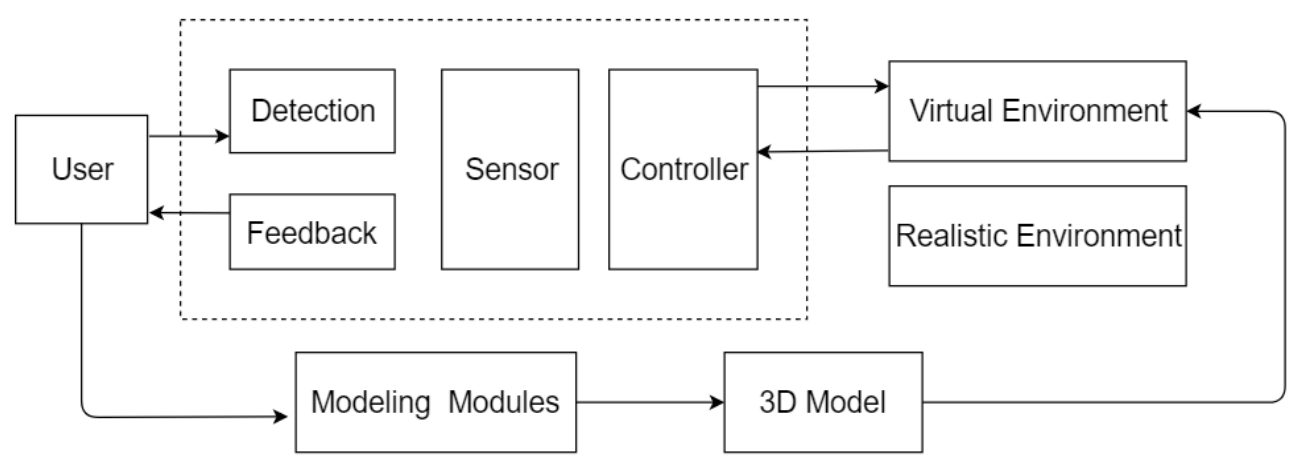

Figure 2. Virtual reality technology system schematic diagram

\section{Conclusion}

In the face of the rapid development of technology, we need to pay more attention to problem-based learning and interaction research in virtual environment, in-depth mining the rich learning value and its reference significance for online education and learning. It has reference value to the structure of network learning resources. At present, the structure of network learning resources mostly stays in the category of individual learning, only focusing on the listing and display of knowledge, less reflecting the problem situation, and it is difficult to stimulate learners' active exploration and learning interaction. Secondly, the problem situation and interaction in network learning is an important means to trigger lasting and in-depth network learning. We need to It is very important to study how to design more problem situations to promote learners to have meaningful interaction, dialogue and consultation, and even to provide the conditions for learners to create and add problem situations, so as to build a dynamic and intelligent online learning environment, which is especially important in today's Open University and open education resources.

\section{Acknowledgments}

Financial support provided by the Humanities and Social Sciences Fund of the Ministry of Education, China(17JAZH100) \& the Special Project on Humanities and art of Xi'an University of Technology (106-451017004).

\section{References}

1. Wang Weiguo, Hu Jinhong. Current situation and development of virtual simulation experiment teaching in foreign universities [J]. Laboratory research and exploration, 2015, 34 (5): 214219

2. Dou Yan, Wang Changwu, Zhao Fengda, et al. Multimodal assessment of hybrid teaching supported by online teaching platform [J]. China education informatization, 2017 (14): 1719
3. Gao Wen. Key conceptions in the learning sciences ( in Chinese) $[\mathrm{M}]$. Shanghai: East China Normal University Press: 17.2009

4. Heim , Michael. The metaphysics of virtual reality ( in Chinese) $[\mathrm{M}]$.Translated by Liu Gang.Shanghai: Shanghai Scientific \& Technical Publishers:79.2000

5. Cheng Haili, Zhang Jingzong, Wang Xianyun, et al. On the principle of "virtual reality combination" in the construction of virtual simulation experiment teaching center $[\mathrm{J}]$. Gansu science and technology.2017, 33 (3): 50-53

6. Xu Xiaofei. Research on digital matting algorithm [D]. Xi'an: Xi'an University of technology. 2009

7. Ning Fangli, Wang Lin. teaching method of mechanical design experiment based on the combination of virtual and real [J]. Laboratory research and exploration. 2017, 36 (2): 165-167 